\title{
ARTERIES OF THE SPINAL CORD IN THE CAT*
}

\author{
BY \\ PETER BRADSHAW \\ From the Department of Neurology, Columbia University, and the Neurological Institute of the Presbyterian Hospital, \\ New York, N.Y.
}

The purpose of this paper is to describe the pattern of arteries supplying the spinal cord in the cat and to present the results of experimental occlusion of certain tributaries of the ventral spinal artery.

Despite the extensive use of the cat in neurological research, surprisingly little has been written about the circulation through the spinal cord in this animal and the original description by Tauber and Langworthy (1935) made no mention of the relative size or importance of the various segmental anastomoses. Subsequent studies in man by Suh and Alexander (1939), in the monkey by Yoss (1950), and in the rat by Woollam and Millen (1955) have shown that there is a considerable disparity in the size of tributaries of the ventral spinal artery. Thus, in man, there are only six or eight unpaired ventral lateral spinal arteries which are greater than $250 \mu$ in diameter. These are the only vessels which are likely to make any significant contribution to blood flow within the ventral spinal artery. Papers, published in abstract form, by Groat (1945) and Brightman (1956) do not indicate whether these features also obtain in the cat. Furthermore, their technique entailed perfusion of a mixture of formalin, Indian ink, gelatin, and saline into the aorta of the cat and we have found this method unsatisfactory, for the site of injection is too far removed from the vessels of the spinal cord and the uneven consistency of the injection mass does not permit filling of the smaller arteries. Both disadvantages are avoided in the present study.

The second section of this paper is concerned with occlusion of certain segmental tributaries of the ventral spinal artery. Interest has been focused in recent years on the possible rôle of ischaemia in the production of lesions of the spinal cord and in particular on the myelopathy associated with cervical spondylosis. Mair and Druckman (1953) described the pathology of two patients with cervical

*This work was completed during the tenure of a travelling fellowship in neurology awarded by the Board of Governors of the General Infirmary at Leeds.

Present address: The Royal Hospital Annexe, Fulwood Road, Sheffield 10. spondylosis and a case of myelopathy which came $\mathbb{\otimes}$ to necropsy. They concluded that the location of 0 the spinal cord lesions favoured insufficiency of the ventral spinal artery as the underlying cause. In $\vec{\circ}$ 1957, I made a survey of 32 patients with osteo- $\overrightarrow{\vec{\omega}}$ arthritis of the cervical spine who were submitted to $\stackrel{\omega}{\omega}$ cervical laminectomy and found no evidence to $\overline{\overline{7}}$ support the contention that osteophytic ridges had caused pressure either upon the ventral spinal artery ${ }_{C}$ or upon the spinal cord (Bradshaw, 1957). However, owing to the limits of the operative field, it wa N impossible to exclude compression of the radiculat arteries, which are tributaries of the ventral spina artery, within the intervertebral foramina either bo osteophytes or fibrosis of the meningeal root sleever Suh and Alexander (1939) have shown that there are $\Phi$ only two or three large segmental tributaries of the ventral spinal artery in the cervical region, and the $\frac{\Phi}{-}$ development of a myelopathy in patients wit $\overrightarrow{0}$ cervical spondylosis might depend on whether one on of these critical arteries had been compressed. Indeed, the lack of correlation between the osteophytic ridges as shown by myelography and the level of the clinical neurological deficit suggests that the cause of the myelopathy is not uncomplicated cord compression.

It seemed important, therefore, to determine by $\overrightarrow{\bar{O}}$ animal experiment whether it is possible to provoke 3 an ischaemic myelopathy by occluding one of the larger ventral lateral spinal arteries or its parent vessel, the radicular artery.

A preliminary study was made of the spinal circulation of the gibbon and rhesus monkeys and of the dog. Though, in these animals, there was a considerable disparity in the size of the ventral 8 lateral spinal arteries and asymmetry of their distribution, there was also a marked individual 으 variation in the levels at which the larger segmental anastomoses occurred. This variation made it impossible to occlude a large radicular artery by a selective operation.

The present study of the pattern of spinal arteries $N$ in the cat showed that though the segmental tribu- N 
taries of the ventral spinal artery in the cervical region were approximately equal in size, this pattern did not obtain in the thoracic and lumbar regions. A very large ventral lateral spinal artery regularly accompanied the fourth lumbar nerve root on the left side and, for this reason the radicular arteries between L. 2 and L. 6 were occluded in the cat.

\section{Material and Methods}

Injection of Spinal Arteries with Latex Solution.-The thorax was opened under nembutal anaesthesia in eight cats, the pericardium incised, and a plastic cannula introduced into the left cardiac ventricle. The cannula was secured in position with artery forceps and the right auricular appendage was slit open. Exsanguination was carried out by the perfusion of $500 \mathrm{ml}$. normal saline through the cannula from a gravity bottle at a height of three feet. When this had been done, the cannula was reconnected to a pressure bottle containing $400 \mathrm{ml}$. of $10 \%$ formaldehyde (unneutralized) and this was perfused at a pressure of $150 \mathrm{~mm}$. Hg.

Major arteries within the thorax were identified and exposed by blunt dissection and three ligatures were tied as follows: the first around the ascending arch of the aorta and the second and third around the beginnings of the innominate and subclavian arteries respectively. Distal to each ligature a short length of polyethylene tubing was introduced into the arterial lumen and tied in position. Care was taken to avoid pushing the tubing beyond the left common carotid branch of the innominate artery, the vertebral branch of the left subclavian artery, and the first intercostal branch of the aorta respectively.

Latex solution becomes sticky in contact with glass and solidifies in an acid medium and therefore it is necessary to use an all-metal syringe, previously washed clean of formalin. The syringe was connected in turn to each of the three lengths of polyethylene tubing and the following amounts of latex injected: aorta, 25 to $35 \mathrm{ml}$., innominate, 7 to $12 \mathrm{ml}$., left subclavian 5 to $10 \mathrm{ml}$. Pressures applied to the plunger of the syringe were not measured; usually sufficient latex had been injected into the aorta when filling of small arteries over the bladder wall became visible.

The extradural course of the relevant arteries was studied by dissection, and the brain, spinal cord, and proximal portions of nerve roots, together with their meningeal coverings, were removed in the usual manner. The pattern of the spinal arteries was determined and the diameters of the injected vessels measured under a dissecting microscope.

In four animals the above procedure was modified as follows. After perfusion with formaldehyde a laminectomy, limited to two adjacent vertebrae, was done and a single pair of nerve roots exposed extradurally within the spinal canal. A ligature was tied tightly about one pair of nerve roots to occlude the radicular arteries. Latex was then injected as before.

Injection of Spinal Arteries with Vinyl-lead-acetone.Techniques for the preparation, injection, and subsequent radiography of vinyl-lead-acetone have been described by Kaplan $(1953,1956)$. In the present study three cats were used and the initial procedures for exsanguination and formaldehyde perfusion have already been described.

The injection mass was prepared by shaking $6 \mathrm{~g}$. of vinylite powder in $60 \mathrm{ml}$. of acetone until a solution had been made. Two parts of this mixture were then added to three parts of a liquid lead compound, known as " 126 " (National Lead Company, Brooklyn), while the latter was thinned by heat on a water-bath. The surface precipitate that formed was discarded and a part of the remainder was injected through a fine metal cannula into the vertebral arteries. It was necessary to apply considerable force to the plunger of the syringe to fill the small intrinsic vessels of the spinal cord from the vertebral arteries and, as the solution solidifies rapidly, the whole procedure had to be completed within a few minutes. The spinal cord was removed and radiographed using a $3 \mathrm{~mm}$. focal spot, a $50 \mathrm{Kv}$., $10 \mathrm{M}$.A. $x$-ray tube, and an exposure time of one second. In addition, radiographs were taken of transverse slices of spinal cord 1 to $3 \mathrm{~mm}$. in thickness, using a $0.5 \mathrm{~mm}$. focal spot, $1 \mathrm{Kv}$., 45 M.A. $x$-ray tube, and an exposure time of $\frac{1}{2}$ second. Type " $M$ " industrial film and cardboard cassettes were employed and the negatives were enlarged and printed on high-contrast paper.

Division of Pairs of Radicular Arteries in Live Animals. - Seventeen adult female cats were used. Under nembutal anaesthesia and with an aseptic technique, a longitudinal skin incision was made over the spines of the appropriate vertebrae, the deep fascia incised, and the posterior spinal muscles scraped of their bony attachments. The spinous processes and central portion of two adjacent laminae were removed. Epidural fat was teased away with blunt forceps in order to minimize venous haemorrhage before extending the laminectomy to its full lateral extent. The appropriate pair of lumbar nerve roots was identified and divided extradurally within the spinal canal. Any resultant bleeding was controlled by the temporary application of cotton strips between the dura and the wall of the bony canal. The deep fascia and skin were closed in two layers and the wound sealed with a collodian dressing. Intramuscular penicillin, 300,000 units daily, was given for the first two postoperative days.

The single pairs of lumbar nerve roots divided were as follows:

\begin{tabular}{c|c}
\hline Number of Animals & Pair of Nerve Roots Divided \\
\hline 2 & L. 2 \\
3 & L.3 \\
4 & L.4 \\
4 & L.5 \\
2 & L.6 \\
\hline
\end{tabular}

In two other cats the fourth pair of lumbar nerve roots was exposed but left intact to serve as controls.

Animals were examined daily after operation for evidence of cutaneous sensory loss to pin prick, retention of urine, the placing responses of the hind limbs, the deep tendon reflexes, the pinch reflexes, and gait. Twelve animals were sacrificed by exsanguination and perfusion with $10 \%$ formalin under nembutal anaesthesia on the 14th post-operative day; of the remainder, four (that contracted distemper) on the eighth, and one (with a 
wound infection) on the ninth post-operative days. The whole spinal cord was removed within an hour of death, the presence of the lesion was confirmed, and the spinal canal inspected to exclude vertebral subluxation, disc herniation, infection, and haematoma formation. Sections of the spinal cord were examined macroscopically before they were embedded in paraffin or celloidin. Serial sections were taken from the segment of cord adjacent to the root section and two out of every 10 were stained alternatively with Nissl and Mahon's stains. Four sections were cut from each of the two segments rostral and caudal to the root section and stained with Marchi and Mahon's methods; four other sections were taken from both the mid-thoracic and the lower cervical regions and stained with Mahon's stain. All sections were $20 \mu$ thick.

\section{Results}

Extrinsic Arteries of Spinal Cord.-The vertebral arteries arose from the first part of the subclavian arch at approximately the same level on either side, ascended to the transverse processes of the sixth cervical vertebra, traversed the vertebral foramina, and, adjacent to the ventral surface of the first cervical nerve roots, pierced the meningeal root sleeves to enter the subarachnoid space. After giving off meningeal branches to the posterior fossa, they continued in a rostral and medial direction to the lower border of the pons where they united to form the basilar artery. The subarachnoid portions of the vertebral arteries were 550 to $1,050 \mu$ in diameter. Opposite each intervertebral foramen, between C.2 and C.7, the vertebral arteries gave off lateral branches, the vasa nervorum and arteries to neighbouring muscle, and a medial branch which entered the intervertebral foramen as the radicular artery. Caudal to C.7 the radicular arteries were derived from the intercostal, lumbar, and sacral arteries. In all regions the radicular arteries divided into a dorsal and ventral branch which accompanied the sensory and motor roots respectively. These are the lateral spinal arteries.

The ventral lateral spinal arteries provided branches to the ventral spinal dura and the vertebral bodies and continued in a rostral and medial (sometimes a caudal and medial) direction as tributaries of the ventral spinal artery. Their junction with that vessel was either direct or via a number of short branches. Not all of the ventral lateral spinal arteries were of the same size. In the cervical region (Figs. 1 and 2), between C.3 and C.8 their diameters were approximately equal, with occasional exceptions, and measured 120 to $280 \mu$. At C.2 the arteries were small, 10 to $40 \mu$. In the thoracic and lumbar regions (Fig. 3) there was considerable variation between individual specimens and asymmetry of arrangement. Thers were only eight to

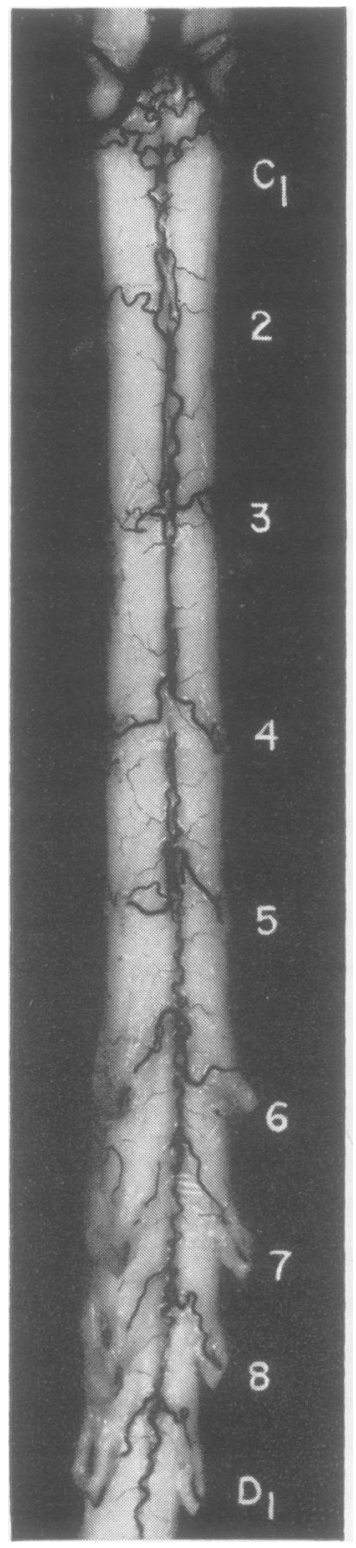

Fig. 1.-Ventral aspect of cervical cord injected with latex. Instead of a ventral spinal artery there are several small parallel vessels. The rostral limit, formed by descending branches from the vertebral arteries, can be seen. The ventral lateral spinal arteries are small at C. 2 on both sides and at C. 3 on the right.

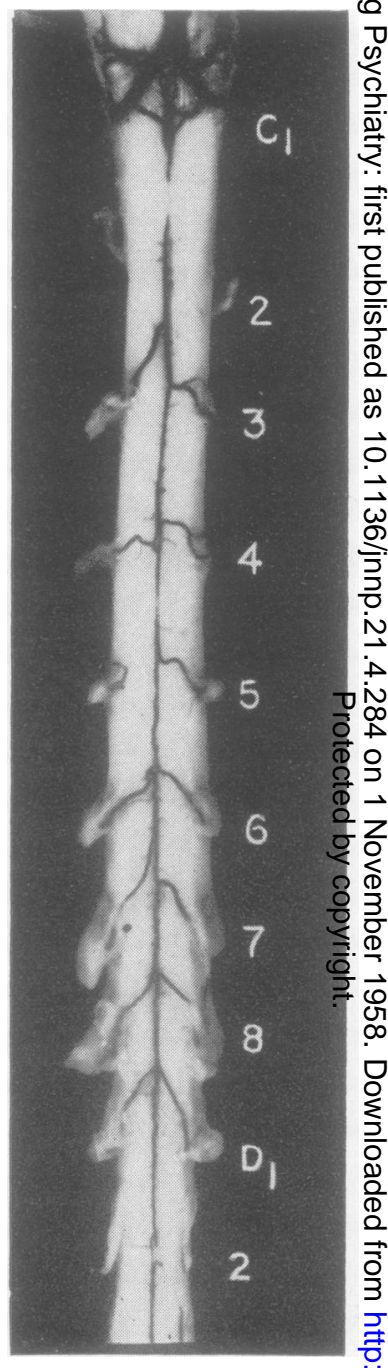

FIG. 2.-Ventral aspect of cervical cord injected with latex. The ventral spinal artery is narrowest at C.2 and the symmetry of the segmental tributaries can be seen. 


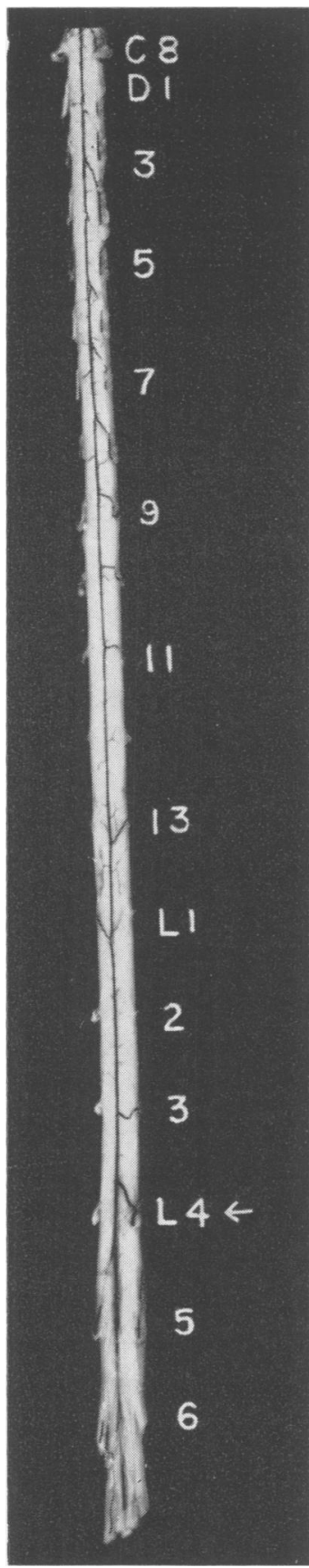

Fig. 3.-Ventral aspect of thoracic and lumbar cord injected with latex, showing the asymmetry of the segmental arteries. Most of the large ventral lateral spinal arteries are on the left ; a very large one is visible at L.4.

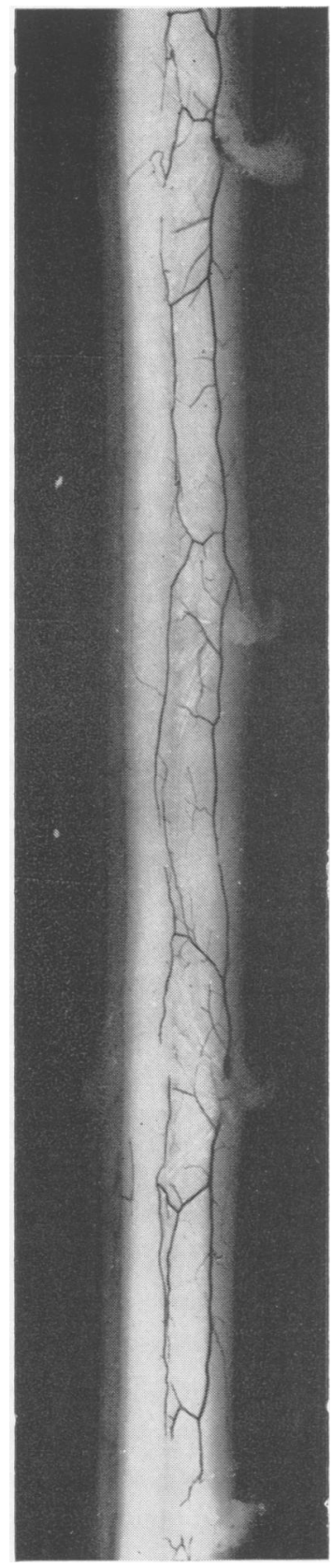

FIG. 4.-Dorso-lateral aspect of thoracic cord injected with latex. One of each pair of the medial and lateral dorsal spinal arteries can be seen on the right.
14 unpaired ventral lateral spinal arteries in the thoracic and three to five in the lumbar region which were greater than $100 \mu$ in diameter; the remainder were very small, 30 to $60 \mu$. Furthermore, the majority of the larger vessels were located on one side, usually the left. A feature constant to all specimens examined was a large artery, 250 to $300 \mu$ in diameter, at L.4 on the left side.

The dorsal lateral spinal arteries gave off branches to the dorso-lateral spinal dura and continued on the ventral surface of the sensory nerve roots as far as the dorsal root entry zone and there divided into ascending and descending branches which anastomosed to form the lateral pair of dorsal spinal arteries (Fig. 4). Diameters of the dorsal lateral spinal arteries were 25 to $150 \mu$ (average $60 \mu$ ) and there was a considerable variation in the distribution of the larger vessels in different specimens. In general, the larger ones were found in segments where the ventral lateral spinal artery was small, for example, at C.2.

The dorsal spinal arteries were small in calibre and in no single specimen was adequate injection obtained throughout the whole length of the spinal cord. Caudal to C.5-7, there were four dorsal spinal arteries (Fig. 4), namely, a lateral pair, formed by the anastomosis of ascending and descending branches of the dorsal lateral spinal arteries, and a medial pair which arose as branches of the former. The lateral pair were 50 to $100 \mu$ diameter and lay at the level of the dorsal root entry zone often covered by the most dorsal fibres of the sensory roots; the medial pair were 25 to $50 \mu$ in diameter and lay in the dorsolateral fissures. Between C.5 and C.7 each of the medial pair of dorsal spinal arteries was seldom a continuous structure and was represented by branches which arose from the lateral pair and which passed in an irregular fashion over the dorsal aspect of the spinal cord. Rostral to C.2, numerous small branches from the vertebral arteries encircled the cord from its ventral aspect and supplied the region of the dorsal columns. From each of the four dorsal spinal arteries and from the ventral spinal artery there were circumferential branches which formed a continuous arterial ring around the spinal cord, and from the lateral pair of dorsal spinal arteries there arose branches which penetrated into the substance of the dorsal horns.

The ventral spinal artery extended from the level of the foramen magnum to the conus medullaris and lay superficially in the ventromedian sulcus though, occasionally, for a few segments, it dipped deeper into that space. In some specimens it was represented by several small and parallel vessels. The rostral limit was formed by the junction of several descending branches of the vertebral arteries, 


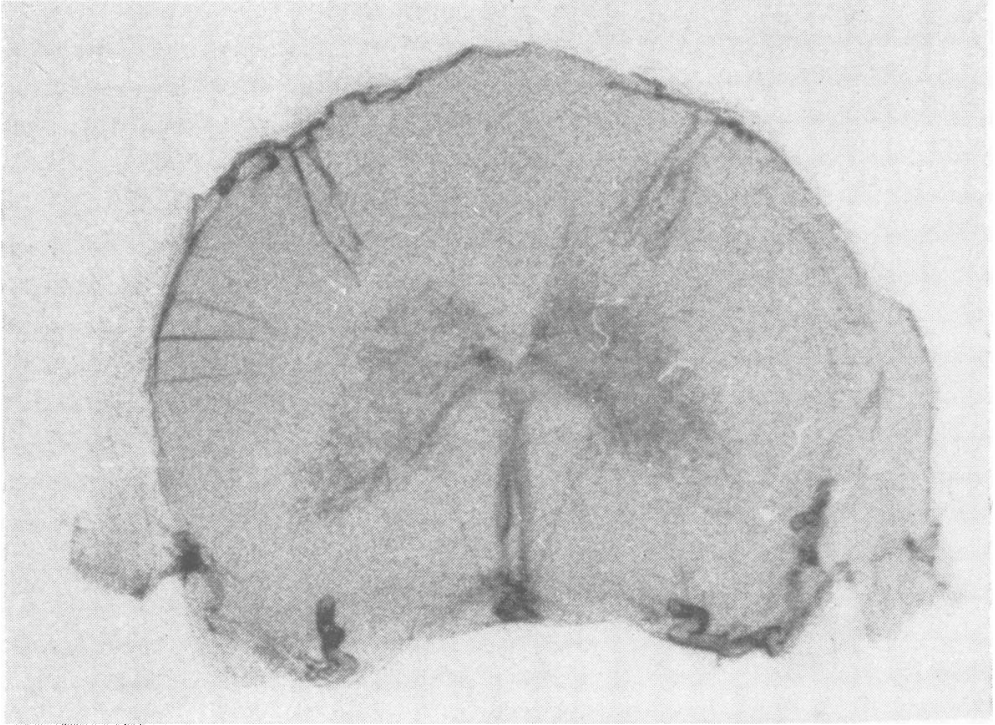

Figs. 5 and 6.-Enlarged radiographs of slices of cervical cord injected with vinyl-lead-acetone. The bilateral distribution of a median sulcal branch to supply the spinal grey matter can be seen, together with some of the circumferential and penetrating branches.

whereas its caudal extent was continuous with the lower sacral arteries. The largest diameters were 300 to $425 \mu$ in the regions of the spinal enlargements and the smallest were 100 to $185 \mu$ in the thoracic region and at the level of the second cervical segment. Throughout its course it was reinforced by segmental tributaries, the ventral lateral spinal arteries. Between each pair of these tributaries there were three to nine circumferential branches which were asymmetrically arranged and passed around the ventro-lateral surface of the spinal cord to anastomose with those from the dorsal spinal arteries.

Other branches of the ventral spinal artery passed into the ventro-median sulcus.

Intrinsic Arteries of the Spinal Cord.--There were two to four unpaired branches of the ventral spinal artery in each spinal segment which entered the ventro-median sulcus and pierced the white spinal commissure. At levels slightly ventral to the central canal, or on either side of it, these median branches divided into a fine arterial network which was distributed throughout the ventral and lateral grey matter (Figs. 5 and 6). Larger branches entered the base of the dorsal horns where they anastomosed with penetrating branches from the lateral pair of dorsal spinal arteries; this intramedullary anastomosis occurred from one to three times in each cervical segment. The white matter, by contrast, had a much poorer blood supply from small penetrating branches which arose from the circumferential anastomotic ring. Such vessels were small in diameter and it is probable that many failed to fill with the injection mass, but they seemed to be most numerous in the region of the lateral columns.

Occlusion of Radicular Arteries before Injection of Latex.-The symmetrical arrangement in the cervical region of the tributaries of the ventral spinal 
artery suggested that blood flow within that artery would not be materially lessened by ligation of any single pair of radicular arteries, with the possible exception of that at C.3. It will be recalled that there is no large ventral lateral spinal artery at C.2 so that ligature of the segmental anastomoses at C.3 might impair circulation in the rostral part of the cervical cord. However, ligation of the single separate pairs of radicular arteries at C.3, C.4, and C.6 and, in the lumbar region, of L.4, did not lead to a filling defect in the ventral spinal artery when latex solution was subsequently injected.

Division of Pairs of Lumbar Radicular Arteries in the Live Cat.-Following the division of single pairs of lumbar nerve roots, together with the radicular arteries that accompanied them, most of the 17 animals subjected to these operations exhibited a neurological deficit. The placing responses were impaired or lost in 12 , and in nine of these the state of the deep tendon reflexes in the hind limbs was also abnormal by reason of inequality, exaggeration, or loss. In addition, these cats walked with their hind limbs in a position of partial flexion and were unsteady on their feet. None of these abnormalities persisted for more than four or five days and the severity and duration of the disturbances were unrelated to the level of the root section. Since these phenomena also occurred in the two controls, in which a pair of nerve roots had been exposed but left intact, it is probable that they were caused by operative trauma to the spinal cord rather than by occlusion of the radicular arteries. When these abnormalities had disappeared, it was possible to detect weakness, limited to muscles innervated by those nerve roots which had been sectioned. At the end of seven to 10 days the disability had become minimal and did not limit activity.

Histological examination of the spinal cords failed to reveal any signs of ischaemic myelopathy. Certain minor changes which were seen included a reduction in the number of ventral horn cells at the level of the lesion and areas of partial demyelination around the circumference of the spinal cord at the same level.

These results show that surgical division of single pairs of lumbar radicular arteries in the cat, including the parent vessel of the large ventral lateral spinal artery at L.4 on the left, does not cause an ischaemic myelopathy. In the cat, at least, blood flow within the ventral spinal artery is sufficient to maintain normal structure and function of the spinal cord when a single pair of its segmental tributaries is occluded.

\section{Summary and Conclusions}

The extrinsic arteries of the spinal cord have been studied in the cat after the injection of latex into the left subclavian and innominate arteries and the thoracic aorta. Though the general arterial pattern resembles that which has been reported for man there are important differences.

(1) Segmental tributaries of the ventral spinal artery in the cat are approximately equal in size between C.3 and C.8; in man there are two or three large unpaired tributaries and the remainder are small.

(2) In the thoracic and lumbar regions these segmental tributaries are unequal in size and the larger vessels are situated predominantly on one side in both the cat and man, but large tributaries are more numerous in the cat.

(3) There is a large ventral lateral spinal artery on the left side at L. 4 which was constantly present in all of the 10 cats examined.

(4) The spinal dura is more vascular in the cat than in man.

The intrinsic arteries of the spinal cord have been studied in the cat by radiology after the injection of vinyl-lead-acetone into the vertebral arteries. Features which distinguish the pattern of spinal arteries in the cat from that reported for man include the following:

(1) Median branches of the ventral spinal artery, which enter the ventro-median sulcus and pierce the white spinal commisure, each supply the grey matter on both sides whereas in man it is reported that each branch has a unilateral distribution.

(2) Within the dorsal horns there is an extensive anastomosis between the ventral and dorsal spinal arteries which has not been described in man.

The ligation of single pairs of radicular arteries in the cervical region and at L.4 in the cat does not prevent subsequent filling of the ventral spinal artery with latex. When single pairs of lumbar radicular arteries are severed in the living cat there are no permanent sequelae to suggest an ischaemic myelopathy and when these animals are sacrificed at 14 days the gross histology of the spinal cord is normal.

\section{REFERENCES}

Bradshaw, P. (1957), Quort. J. Med., 26, 177.

Brightman, M. W. (1956). Anat. Rec., 124, 264.

Groat, R. A. (1945). Ibid., 91, 278 .

Kroat, R. A. (1945), Ibid., 91, 278.

Kaplan, H. A. (1953). Ibid., 116, 507. 364.

Mair, W. G. P., and Druckman, R. (1953). Brain, 76, 70.

Suh, T. H., and Alexander, L. (1939). Arch. Neurol. Psychiat. (Chicago), 41, 659.

Tauber, E. S., and Langworthy, O. R. (1935). J. nerv. ment. Dis., 81, 245 .

Woollam, D. H. M., and Millen, J. W. (1955). J. Neurol. Psychiat.. $18,97$.

Yoss, R. E. (1950). Univ. Mich. med. Bull., 16, 333. 